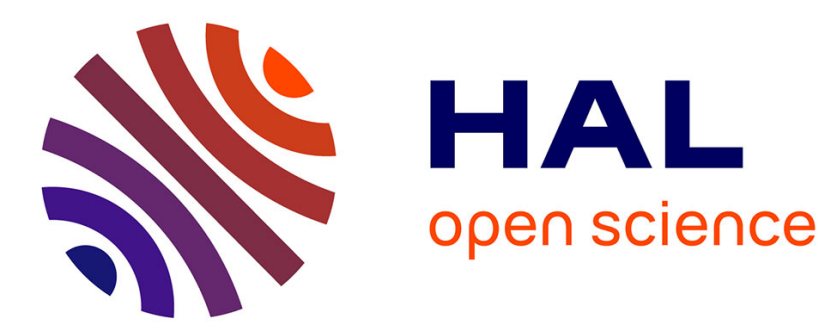

\title{
Linear demosaicing inspired by the human visual system
} David Alleysson, Sabine Süsstrunk, Jeanny Hérault

\section{To cite this version:}

David Alleysson, Sabine Süsstrunk, Jeanny Hérault. Linear demosaicing inspired by the human visual system. IEEE Transactions on Image Processing, 2005, 14 (4), pp.439-449. 10.1109/tip.2004.841200 . hal-00204920

\section{HAL Id: hal-00204920 \\ https://hal.science/hal-00204920}

Submitted on 15 Jan 2008

HAL is a multi-disciplinary open access archive for the deposit and dissemination of scientific research documents, whether they are published or not. The documents may come from teaching and research institutions in France or abroad, or from public or private research centers.
L'archive ouverte pluridisciplinaire HAL, est destinée au dépôt et à la diffusion de documents scientifiques de niveau recherche, publiés ou non, émanant des établissements d'enseignement et de recherche français ou étrangers, des laboratoires publics ou privés. 


\title{
Linear Demosaicing inspired by the Human Visual System
}

\author{
David Alleysson, Sabine Süsstrunk, Member, IEEE and Jeanny Hérault
}

\begin{abstract}
There is an analogy between single-chip color cameras and the human visual system in that these two systems acquire only one limited wavelength sensitivity band per spatial location. We have exploited this analogy, defining a model that characterizes a one-color per spatial position image as a coding into luminance and chrominance of the corresponding three-colors per spatial position image. Luminance is defined with full spatial resolution while chrominance contains sub-sampled opponent colors. Moreover, luminance and chrominance follow a particular arrangement in the Fourier domain, allowing for demosaicing by spatial frequency filtering. This model shows that visual artifacts after demosaicing are due to aliasing between luminance and chrominance and could be solved using a pre-processing filter. This approach also gives new insights for the representation of single-color pe $r$ spatial location images and enables formal and controllable procedures to design demosaicing algorithms that perform well compared to concurrent approaches, as demonstrated by experiments.
\end{abstract}

Index Terms- Color, Demosaicing, Fourier analysis, Linear filtering.

\section{INTRODUCTION}

A color image usually consists of three channels per pixel, each carrying the information of a specific wavelength sensitivity band (red, green, or blue) to allow for color processing and display. Intuitively, one would assume that acquiring such an image requires cameras with three spatially aligned sensors, each preceded by a different color filter to capture the information for a given part of the visible spectrum. However, to reduce size, cost, and image registration errors, most digital cameras only have a single sensor with a color filter array (CFA) placed in front of it. Consequently, only one spectral band-or one color-is captured at each spatial location. Such an arrangement can also be found in the human visual system (HVS), in particular in the fovea, where three types of cones called $\mathrm{L}$

Manuscript received May 26, 2003.

D. Alleysson was with LCAV/EPFL. He is now with the Laboratory for Psychology and NeuroCognition (LPNC), University Pierre-Mendes France, Grenoble (David.Alleysson@upmfgrenoble.fr). Ph. (+33)476825893. Fax (+33)476827834.

S. Süsstrunk is with the Audiovisual Communications Laboratory (LCAV), Ecole Polytechnique Fédérale de Lausanne (EPFL) (Sabine.Susstrunk@epfl.ch).

J. Hérault is with Laboratory of Image and Signal (LIS), Institut National Polytechnique de Grenoble (INPG) (Jeanny.Herault@lis.inpg.fr). for Long, $\mathrm{M}$ for Middle and $\mathrm{S}$ for Short wavelength band sensitivity are arranged in a single lattice. Thus, CFA-based cameras and the human retina share the property that they sample only one color per spatial location. Because singlechip cameras and cone lattices do not sample luminance and chrominance information separately, luminance being defined as a spatial map of intensity and chrominance as a map of chromatic components, spatial and chromatic information is mixed together in a unique bi-dimensional lattice after sampling. To provide a pleasing image from this mixture, some "processing" needs to be applied. In cameras, a color demosaicing (also called demosaicking) algorithm is used to reconstruct three wavelength measurements per spatial position. In this paper, we investigate how it is possible to identify and separate both spatial and chromatic information using luminance and chrominance in the sampled image, and what the conditions are for this color image representation to be lossless.

It is well known that achromatic (luminance) spatial acuity in the HVS is better than chromatic spatial acuity [1]. Moreover, it has been found experimentally that trichromacy does not influence spatial acuity [2]. The ability of a trichromatic human observer to detect achromatic gratings is similar to those theoretically given by a retina composed of only one type of photoreceptor. This suggests that the spatial information obtained by the retinal sampling is preserved, and that single-chromatic information per spatial location is sufficient for spatial and color vision. However, demosaicing algorithms applied to CFA images generate artifacts, characterized mostly by blurring and false color, which reduce the spatial resolution and color accuracy of the reconstructed image. It appears that the HVS has found a solution to reconstruct this subsampling with minimal loss. This poses the question if this property of the HVS could be imitated for the case of digital images acquired through a CFA.

To answer this question, we have developed a formalism of the inverse problem and defined the operations that transform a three-color per pixel image into a single-color per pixel image. We found that a single-color per pixel image could be interpreted as an image representation where luminance and chrominance have different locations in the Fourier domain [3, 4]. This allows the design of a demosaicing algorithm that uses estimators in the Fourier domain to reconstruct both luminance (spatial information) and chrominance (chromatic information). Luminance is coded in its entirety, whereas chromatic information is sub- 
sampled. Moreover, this formalism allows us to understand artifacts in the reconstruction as aliasing between luminance and chrominance, and explain why the Bayer CFA is the most optimal spatial arrangement of three color samples on a square grid.

The paper is organized as follows. First, we present a simple case of a CFA camera design to show how to build an alias-free system in the case of demosaicing by bilinear interpolation. We then discuss a model of spatio-chromatic sampling that illustrates the properties of images with only one color per spatial location. We describe our demosaicing algorithm and the optimal filter for the best reconstruction. Finally, we review the principal concurrent algorithms of color demosaicing and compare some of them to our method.

\section{EXACT DEMOSAICING: A SIMPLE CASE}

An image is a sampled version of the real world in the sense that the energy of the original scene is only known at discrete positions. The sampling operation generates aliasing if the sampling frequency of the sensor is not high enough compared to the maximum frequency of the captured scene. When aliasing occurs, the original signal cannot be reconstructed without errors. According to the Whittaker-Shannon sampling theorem, when the sampling frequency is at least twice the maximum frequency of the continuous signal, there will be no aliasing and the original may be recovered exactly. This is discussed in $[5,6]$ for the case of CFA images. To reconstruct the original signal, a sinc function should be used for the interpolation. Unfortunately, the support of this function is infinite and slowly decaying, and therefore not usable in practice. An approximation of this ideal interpolation function is often used [7].

Natural scenes do not have a fixed frequency limit that allows designing a corresponding sampling frequency of the sensor. However, the modulation transfer properties of the optics of the camera act as a low pass filter and determine the cut-off spatial frequency of the captured image. To avoid aliasing, the optimal design of a camera is thus to match the optics with the sampling frequency of the sensor. This is easily accomplished for a monochromatic imager or a three-sensor imager because the sampling frequency is unique. For CFA imagers, however, the sampling frequency of one of the three colors is different from the other two because it is impossible to arrange three colors on a square grid with three identical (horizontal and vertical) sampling distances, as shown in Figure 1. The arrangement of colors on the CFA also determines the subsequent interpolation procedure. Thus, the design of a CFA camera is a compromise between optical and interpolation constraints. To help solve this problem, Greivenkamp [8] proposes an optical filter called birefringent filter that allows wavelength dependent optical processing to match the optical blur with any configuration of colors in the CFA. Also, Weldy [10] proposes a design of a CFA for which the interpolator could be optimized for the optical filter used.

The most commonly used CFA is called Bayer CFA after the name of its inventor [11]. As shown in Figure 1, it consists of alternating red and green pixels on odd lines and green and blue pixels on even lines. In Section III-C, we will show that the Bayer CFA is the most optimal spatial arrangement of three color filters in terms of avoiding aliasing. This arrangement minimizes artifact generation in general, independently of the algorithm used for demosaicing. Note that the framework we propose is not restricted to this type of CFA, but since its arrangement is optimal we will use it for the rest of the paper.

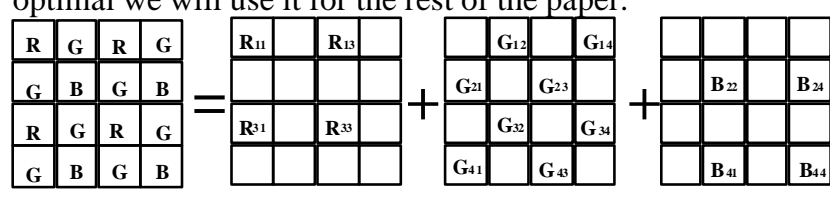

Figure 1. Decomposition of the Bayer CFA into three sub-sampled lattices.

Let us consider the design of a camera with a Bayer CFA. For this example, we use a simple algorithm to interpolate, namely the bilinear interpolation, instead of the ideal sinc interpolator. In reference to Figure 1, to interpolate the red pixel $R$ at position $(2,2)$ and position $(3,2)$ in the grid, we apply:

$R_{22}=\frac{R_{11}+R_{13}+R_{31}+R_{33}}{4} \quad R_{32}=\frac{R_{31}+R_{33}}{2}$

The bilinear interpolation depends on the position of the missing pixel, and differs also for the green pixels with respect to red and blue. Due to the regularity of the CFA pattern, it is possible to define convolution kernels that apply in each color channel separately. The following filter (eq. 2) can easily be implemented in a camera-internal processor, such as a digital signal processor (DSP), to compute the bilinear interpolation efficiently.

$F_{R, B}=\left[\begin{array}{ccc}1 & 2 & 1 \\ 2 & 4 & 2 \\ 1 & 2 & 1\end{array}\right] / 4 \quad F_{G}=\left[\begin{array}{ccc}0 & 1 & 0 \\ 1 & 4 & 1 \\ 0 & 1 & 0\end{array}\right] / 4$

$F_{R_{B}}$ is the bilinear interpolation filter for the red and blue channels, and $F_{G}$ is the interpolation filter for the green channel. To perform bilinear interpolation, these convolution filters apply directly on the corresponding red, green, or blue channel where missing pixels are first filled with zeros.

In Figure 2, we illustrate the reconstructed color image, using the bilinear interpolation filters of eq. 2. The original three-colors per pixel image (Fig. Za) was sub-sampled according to the Bayer CFA. The interpolated image (Fig. 2b) shows two artifacts inherent to demosaicing: blurring and the generation of false color, also called color aliasing. Topfer et al. [5] have already discussed these two artifacts generated by bilinear interpolation demosaicing. They showed that blurring and false color could be fully explained when considering the Fourier representation of sub-sampled color signals and interpolation filters.

These artifacts are a consequence of violating the requirements dictated by the sampling theorem. In this example, we have implicitly assumed that optical blurring is 
designed for a monochromatic or three-sensor camera because we have applied sub-sampling on the original three colors per pixel image. The sub-sampling according to the Bayer CFA therefore reduces the sampling frequency in each color channel, generating a mismatch with the optical behavior of the camera. It is possible to prevent the subsequent aliasing artifacts by applying an anti-aliasing filter before sub-sampling the image. As each channel is sub-sampled by a factor of two in the horizontal and vertical directions, we need to ensure that the maximum of the Fourier spectrum of each channel occupies only half of the total frequency spectrum. This is not exactly true for the green channel where the diagonal directions are not subsampled. However, we consider only the worst case, applying for the green channel the same anti-aliasing filter as for red and blue.
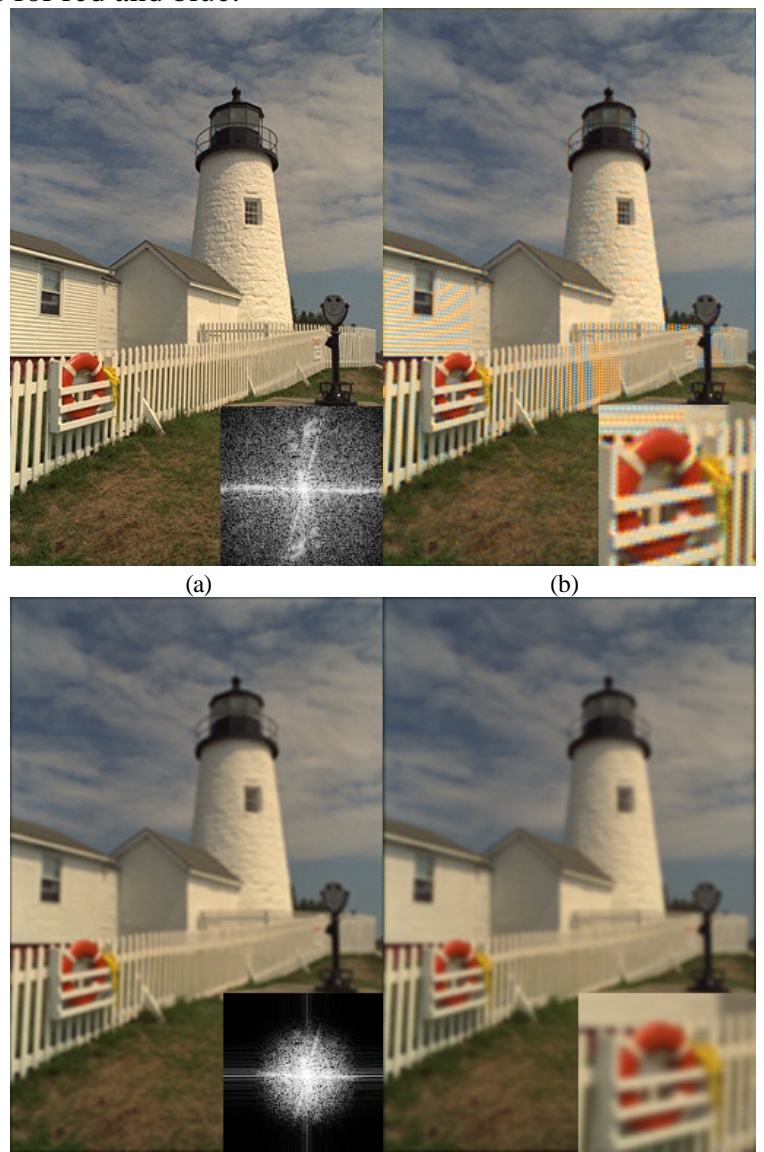

(c)

(d)

Figure 2. Example of a bilinear interpolation computed with convolution filters. (a) Original image: the frequency spectrum of the red (and green and blue) occupies most of the Fourier spectrum. (b) Reconstructed image with bilinear interpolation. (c) Low pass filtering applied to the original image to reduce the size of the Fourier spectrum of the image. (d) The reconstruction of image (c) after mosaicing according to the Bayer CFA and demosaicing by bilinear interpolation shows no artifacts.

Figure 2 illustrates this concept. In a normal three-color per pixel image (Fig. 2-a), each color channel generally has a large Fourier spectrum (Fig. 2-a inset), occupying most of the Fourier space. If we sub-sample such an image, the reconstruction would result in aliasing because the sub- sampling operation replicates the spectrum of the image in each corner and middle of the sides of the Fourier window. Original and aliased signals are mixed and cannot easily be separated. To prevent this, the Fourier spectrum of each color layer should be limited to $1 / 2$ of the sampling frequency. We can ensure this by applying a low-pass filter on each channel of the image (Fig. 2-c). It is then possible to apply mosaicing to the image according to the Bayer CFA so that the bilinear interpolation results in a reconstructed image without any aliasing artifacts (2-d).

In general, the procedure we outlined above to reconstruct an image without visible aliasing is not satisfying. The useful frequency range of the sampled image is half the frequency theoretically provided by the pixel pitch of the sensor. Moreover, the point-spread function that is applied identically on each color channel should be designed for the worst case, i.e. red and blue, which reduces the frequency range of green by half of its actual sampling frequency. For these reasons, this solution is not acceptable and better demosaicing algorithms need to be found.

\section{SPATIAL CHROMATIC SAMPLING}

\section{A. Introduction}

In this section, we describe a model of spatial chromatic sampling that reveals the nature of a CFA image in terms of spatial and chromatic behavior and allows us to design a better schema for interpolation. By examining its properties, we show that a CFA image is composed of the sum of luminance signals carrying achromatic spatial information at full resolution, and opponent color signals carrying chromatic information at lower resolution.

As mentioned above, spatial and chromatic information is mixed together in images resulting from CFA sensors and the human retina, as only one spectral sensitivity per spatial location is available. The retina uses random samples on a hexagonal-like grid, which greatly reduces aliasing [12]. However, this is not feasible in current CFA sensors where the sampling is regular on a periodic grid. Additionally, we know that color information is coded into luminance and opponent color signals at an early stage in the retina to optimize the representation of light information [13]. This has also been described as spatial and temporal multiplexing between luminance and chromatic information at the ganglion cell level of the retina [14]. Therefore, the representation of a color image as luminance and opponent colors better matches human perception than an RGB color encoding. In the particular context of CFA sensors, we can consider each color sample to carry a part of the spatial (intensity) information, due to its position, and a part of the chromatic (wavelength's band) information, due to its spectral sensitivity.

It is important to note that our luminance and opponent color definitions, resulting from mosaiced images, are not directly related to the usual definition of luminance and opponent colors used in image and video processing 
(YCrCb), nor the definitions of luminance and color opponency found in human psychophysics $(\mathrm{V}(\lambda)$, Red/Green, Blue/Yellow). These latter decompositions are based on principal component analysis of RGB or LMS [13]. In an effort to diminish encoding redundancy, RGB or LMS signals can be transformed into a three-dimensional decorrelated luminance and opponent color space. In this study, we will show that sampling a single color per spatial location is equivalent to sampling an achromatic signal (luminance) at each spatial location and a chromatic signal (chrominance) composed of three sub-sampled opponent color signals. Correspondence with other luminance and opponent color spaces is possible by combining two of the three opponent channels.

\section{B. Model of a CFA image}

A color image $I$ can be defined by three color components $C_{i}$ at each discrete spatial location $(x, y)$. This can be expressed as a vector of three dimensions for each pixel.

$$
I=\left\{C_{i}(x, y)\right\}, i \in\{R, G, B\},(x, y) \in \square^{2}
$$

Each color component $C_{i}$ corresponds to the sampling of spatially $(x, y)$ and spectrally $(\lambda)$ variable input irradiance $E(x, y, \lambda)$ through a spectral sensitivity function $\varphi_{i}(\lambda)$ given by the filter and sensor characteristics.

$$
C_{i}(x, y)=\int_{\lambda} E(x, y, \lambda) \varphi_{i}(\lambda) d \lambda
$$

Identically, the same image can also be represented by its luminance and opponent colors. We define our luminance as a scalar intensity image containing achromatic information of the original scene. This signal can be estimated from $C_{i}(x, y)$ using the sum or the mean of the three color components. In fact, any projection in the RGB space with positive combination $p_{i}$ of $C_{i}$ components is suitable. The goal of this estimator is to maximize the ability to detect spatial information independently of the wavelength component of this information to form a spatial map of intensity. The chrominance part can be defined as the remaining three-dimensional vector after we extract the scalar luminance from the original vector. Thus, the color image $I$ can also be represented as a sum of a scalar representing luminance $\Phi$, and a three-dimensional vector representing opponent colors that we call chrominance $\Psi$ in reference to television standards.

$$
\begin{aligned}
& I=\left\{C_{i}(x, y)\right\}=\Phi(x, y)+\left\{\Psi_{i}(x, y)\right\} \\
& \Phi(x, y)=\sum_{i} p_{i} C_{i}(x, y) \\
& \Psi_{i}(x, y)=\left(1-p_{i}\right) C_{i}(x, y)-\sum_{j \neq i} p_{j} C_{j}(x, y) \\
& \sum p_{i}=1, \quad p_{i}>0
\end{aligned}
$$

Thus, each pixel of a color image $I$ can be written as the sum of a scalar and a vector. The scalar part $\Phi$ is composed of the weighted positive sum of each color channel $C_{i}(x, y)$, and the chrominance is a vector composed of three opponent color components $\Psi_{i}$. In this model, the decomposition of a color image containing three components per pixel (i.e. R,G, and B) results in four components. One component, luminance, contains the achromatic intensity information of the color image. When we subtract the luminance from the color image, we obtain chrominance. This chrominance, composed of three chromatic opponent signals, is representative of the chromatic information of the color image independently of the achromatic intensity $\left(\sum \Psi_{i}=0\right)$. Note that these definitions are different than the usual ones, but helpful in the case of a CFA image. Figure 3-abc shows an example of the decomposition of a color image with $p_{R}=p_{G}=p_{B}=1 / 2$.
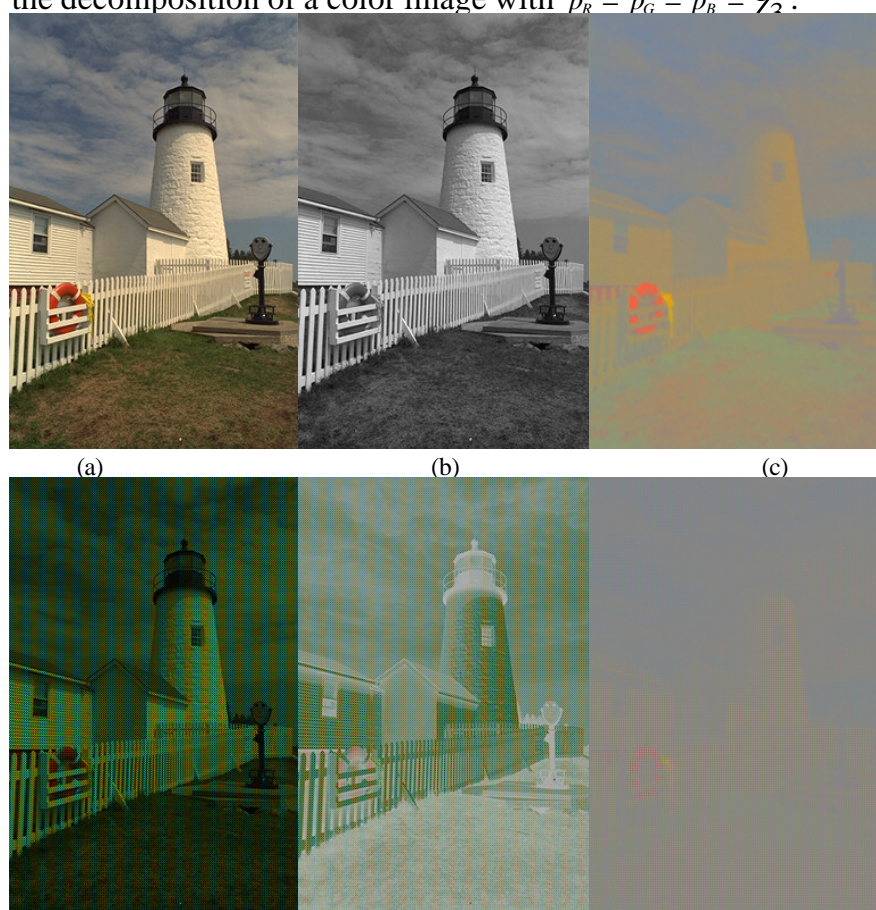

(d)
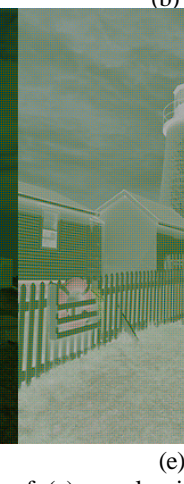

Figure 3: Decomposition of (a) a color image into (b) luminance and (c) chrominance as defined in eq. 5. (d) A CFA image with a single chromatic sensitivity per spatial location according to the Bayer CFA arrangement (the image appears greenish because of the double number of green pixels in the CFA). When subtracting luminance (b) from the CFA image (d), the resulting image is (e), which corresponds to a multiplexed version of sub-sampled chrominance. Selecting pixels in front of each color sample of the Bayer CFA (i.e. de-multiplexing opponent colors) results in image (f). Also, sub-sampling image (c) results exactly in image (f). Thus, image (c) can be recovered from (f) using interpolation

Unlike a regular three-channel color image, a CFA image $I_{C F A}(x, y)$ is already a scalar image having only one color component per spatial location. This can be expressed as a projection of the sub-sampled values on the unity vector [1 $11]$ in the RGB color space [3, 4]:

$I_{C F A}(x, y)=\sum_{i} C_{i}(x, y) m_{i}(x, y)$

$m_{i} \cdot m_{j}=\left\{\begin{array}{ll}1 & \text { for } i=j \\ 0 & \text { for } i \neq j\end{array} \quad, \quad \sum_{i} m_{i}=1\right.$

where $m_{i}(x, y)$ are three orthogonal sub-sampling functions taking values 1 or 0 if the color $i$ is present at discrete location $(x, y)$ or not. For the particular case of the Bayer CFA, $m_{i}(x, y)$ can be written as: 


$$
\begin{aligned}
& m_{R}(x, y)=(1+\cos (\pi x))(1+\cos (\pi y)) / 4 \\
& m_{G}(x, y)=(1-\cos (\pi x) \cos (\pi y)) / 2 \\
& m_{B}(x, y)=(1-\cos (\pi x))(1-\cos (\pi y)) / 4
\end{aligned}
$$

$m_{i}(x, y)$ can also be thought of as modulation functions that realize the spatial multiplexing of sub-sampled color signals. These functions are composed of a constant part plus a modulation part. We can write $m_{i}(x, y)=p_{i}+\tilde{m}_{i}(x, y)$, where $p_{i}$ is constant over space and $\tilde{m}_{i}(x, y)$ is a fluctuation function with zero mean over space. Thus, the CFA image can be expressed as the sum of scalar luminance and scalar chrominance:

$$
I_{C F A}(x, y)=\underbrace{\sum_{i} p_{i} C_{i}(x, y)}_{\Phi(x, y)}+\underbrace{\sum_{i} C_{i}(x, y) \tilde{m}_{i}(x, y)}_{\Psi_{C F A}(x, y)}
$$

Luminance is defined exactly as in eq. 5, while chrominance depends on the modulation function $\tilde{m}_{i}$. The fact that luminance is not multiplied by a modulation functions means that it is defined with full spatial resolution. Thus, luminance is not subject to interpolation rather it can be estimated directly from the CFA image. Theoretically, luminance could be recovered from a CFA image with the same spatial resolution as from an image acquired with three sensors. The idea proposed by Crane et al. [15] to use pre-determined coefficients directly from the CFA image for demosaicing is therefore relevant. But the choice of the predetermined coefficients should be dependent on the optimization of the luminance estimation rather than on the constant hue hypothesis used by them (see Section IV).

While eq. 8 establishes that luminance is exactly present in a CFA image (even if it is subject to aliasing with chrominance), we still need to provide a way to estimate it at each spatial position from the neighboring RGB pixel values of the CFA image. With our definition of luminance as a scalar, it should correspond to a unique projection in RGB space at each position, meaning that the percentage (weights) of red, green, and blue pixel values that compose the luminance estimator should remain constant over all spatial positions. One way to design a luminance estimator is therefore to compute the parameter $p_{i}$ with such a constraint.

Let us assume that we can estimate luminance by a $3 \times 3$ unitary symmetric convolution kernel that has three degrees of freedom, as shown by coefficients $a, b$ and $c$ in eq. 9 . We impose the constraint that the signal resulting from the convolution of the CFA image with this kernel has exactly the same weight of $R, G$ and $B$ at each spatial location. There are three different $3 \times 3$ RGB patterns in the Bayer CFA, which result in two conditions. The third pattern, exchanging the position of blue and red, does not result in an additional condition. The third condition is that the filter is unitary. If $\Phi_{E t}$ is the estimated luminance by a $3 \times 3$ convolution kernel applied to a CFA image, we can write:

$$
\begin{aligned}
& \Phi_{E s t}=\left[\begin{array}{lll}
R & G & R \\
G & B & G \\
R & G & R
\end{array}\right] *\left[\begin{array}{lll}
a & b & a \\
b & c & b \\
a & b & a
\end{array}\right]=\left[\begin{array}{lll}
G & R & G \\
B & G & B \\
G & R & G
\end{array}\right] *\left[\begin{array}{lll}
a & b & a \\
b & c & b \\
a & b & a
\end{array}\right] \Leftrightarrow\left\{\begin{array}{cc}
4 a=2 b & \text { condition for R } \\
2 b=c & \text { condition for B } \\
4 a+4 b+c=1 & \text { norm }=1
\end{array}\right. \\
& \Leftrightarrow\left\{a=\frac{1}{16} b=\frac{1}{8}, c=\frac{1}{4}\right\} \Leftrightarrow \Phi_{E s t}=\frac{R+X+B}{4} \Leftrightarrow\left\{p_{R}=\frac{1}{4}, p_{G}=\frac{1}{2}, p_{B}=\frac{1}{4}\right\}
\end{aligned}
$$

Thus, if the luminance signal is defined as $\Phi_{E s t}=(R+2 G+B) / 4$, the kernel $F_{R, B}$ of eq. 2 allows extracting this signal directly from the CFA image.

In order to clarify the composition of the scalar chrominance signal (eq. 8) in a CFA image, we select color measurements of each photoreceptor type by multiplying by the modulation functions $m_{j}(x, y)$. Note that $\tilde{m}_{i}(x, y)$ can be rewritten as $\tilde{m}_{i}(x, y)=\left(1-p_{i}\right) m_{i}(x, y)-\sum p_{\ell} m_{\ell}(x, y)$.

$$
\begin{aligned}
& \Psi_{C F A}(x, y) m_{j}(x, y)=m_{j}(x, y) \sum_{i} C_{i}(x, y)\left[\left(1-p_{i}\right) m_{i}(x, y)-\sum_{\ell \neq i} p_{\ell} m_{\ell}(x, y)\right] \\
& =m_{j}(x, y)\left[\left(1-p_{j}\right) C_{j}(x, y)-\sum_{k \neq j} p_{k} C_{k}(x, y)\right]
\end{aligned}
$$

Substituting $j$ by $\mathrm{R}, \mathrm{G}$ and $\mathrm{B}$, and $p_{j}$ by the results found in eq. 9 , i.e. $p_{R}=1 / 4 ; p_{G}=1 / 2 ; p_{B}=1 / 4$, chrominance in a CFA image can be expressed as:

$$
\Psi_{C F A}(x, y)=\sum_{j} \Psi_{C F A}(x, y) m_{j}(x, y)
$$

$$
\begin{aligned}
& =\left[\frac{3}{4} C_{R}(x, y)-\frac{1}{2} C_{G}(x, y)-\frac{1}{4} C_{B}(x, y)\right] m_{R}(x, y) \\
& +\left[\frac{1}{2} C_{G}(x, y)-\frac{1}{4} C_{R}(x, y)-\frac{1}{4} C_{B}(x, y)\right] m_{G}(x, y) \\
& +\left[\frac{3}{4} C_{B}(x, y)-\frac{1}{2} C_{G}(x, y)-\frac{1}{4} C_{R}(x, y)\right] m_{B}(x, y)
\end{aligned}
$$

Thus, the scalar chrominance in a CFA image is in fact composed of three sub-sampled and modulated opponent color images, as illustrated in the Figure 3. To recover full chrominance, as defined in eq. 5 , we have to demodulate it by multiplying with the modulation functions $m_{i}$, resulting in a vector of three sub-sampled opponent colors. An interpolation, for example with kernels described in eq. 2, allows reconstructing all pixels of the three opponent color channels.

Note that our definition of luminance and chrominance that allows us to relate a three-color image and a CFA image needs four dimensions: one for luminance and three for chrominance. Also, the weights of R, G and B that compose these signals depend on the proportion of their corresponding filter occurrence in the CFA. In the following section we will show that luminance and chrominance in a CFA image have specific location in the Fourier domain and consequently can be estimated by frequency selection.

\section{Fourier representation}

From the representation of the CFA image $I_{C F A}$ given in eq. 6, we can compute its Fourier transform as the convolution of the Fourier transform of each color layer of the original image $\hat{C}_{i}\left(f_{x}, f_{y}\right)$ and the Fourier transform of the modulation function $\hat{m}_{i}\left(f_{x}, f_{v}\right)$ : 


\section{$\widehat{I}_{C F A}\left(f_{x}, f_{y}\right)=\sum \hat{C}_{i}\left(f_{x}, f_{y}\right) * \hat{m}_{i}\left(f_{x}, f_{y}\right)$}

where $\quad$ represents the Fourier transform, $*$ the convolution operator, $\widehat{C}_{i}\left(f_{x}, f_{y}\right)$ the Fourier transform of the color layer $i$ and $\hat{m}_{i}\left(f_{x}, f_{v}\right)$ the Fourier transform of the modulation function $m_{i}(x, y)$. The modulation functions defined in eq. 7 are based on cosines and have their Fourier transforms expressed as Diracs. Given $f_{r}=f_{x}-r / 2, f_{s}=f_{y}-s / 2$ and $\delta$ the discrete Dirac distribution, they can be expressed as:

$\widehat{m}_{R}\left(f_{x}, f_{y}\right)=\pi^{2}\left(\sum_{r=-1}^{1} \frac{1}{1+|r|} \delta\left(f_{r}\right)\right)\left(\sum_{s=-1}^{1} \frac{1}{1+|s|} \delta\left(f_{s}\right)\right)$

$\widehat{m}_{G}\left(f_{x}, f_{y}\right)=2 \pi^{2} \delta\left(f_{x}\right) \delta\left(f_{y}\right)-\frac{\pi^{2}}{2}\left(\sum_{\substack{r=1 \\ r \neq 0}}^{1} \delta\left(f_{r}\right)\right)\left(\sum_{\substack{s=1 \\ s \neq 0}}^{1} \delta\left(f_{s}\right)\right)$

$\hat{m}_{B}\left(f_{x}, f_{y}\right)=\pi^{2}\left(\sum_{r=-1}^{1} \frac{(-1)^{r}}{1+|r|} \delta\left(f_{r}\right)\right)\left(\sum_{s=-1}^{1} \frac{(-1)^{s}}{1+|s|} \delta\left(f_{s}\right)\right)$

Considering a regular color grid arrangement, these modulation functions localize luminance and chrominance in the frequency domain because $\hat{C}_{i}\left(f_{x}, f_{y}\right) * \delta\left(f_{x}-a, f_{y}-b\right)=\hat{C}_{i}\left(f_{x}-a, f_{y}-b\right)$. Thus, the Fourier spectrum of a spatially multiplexed color image can be expressed as:

$\widehat{I}_{C F A}\left(f_{x}, f_{y}\right)=\sum p_{i} \widehat{C}_{i}\left(f_{x}, f_{y}\right)$

$+\frac{1}{8} \sum_{\substack{r=-1 \\ r \neq 0}}^{1} \sum_{\substack{1=-1 \\ s \neq 0}}^{1} \widehat{C}_{R}\left(f_{r}, f_{s}\right)-\widehat{C}_{B}\left(f_{r}, f_{s}\right)$

$+\frac{1}{16} \sum_{\substack{r=-1 \\ r \neq 0}}^{1} \sum_{\substack{s=-1 \\ s \neq 0}}^{1} \widehat{C}_{R}\left(f_{r}, f_{s}\right)-2 \widehat{C}_{G}\left(f_{r}, f_{s}\right)+\widehat{C}_{b}\left(f_{r}, f_{s}\right)$

Figure 4a shows an example of the amplitude frequency spectrum of a single-color per pixel image sub-sampled according to the Bayer CFA. We clearly see nine regions where energy is concentrated. The center region corresponds to luminance, and the border regions to chrominance.

The frequency localization of luminance and chrominance signals in the Fourier domain allows us to estimate them directly in the CFA by simply selecting corresponding frequency domains. Luminance is estimated by low-pass filtering, while chrominance is estimated by high-pass filtering. The example of the luminance filter given in eq. 9 can be improved by taking into account this frequency localization and by designing a filter with better frequency selection. This will be further discussed in Section IV.

The spatial frequency representation of a CFA image also allows us to clearly formulate the visual artifacts that can arise when reconstructing CFA color images. If the spatial bandwidths of luminance and chrominance are too wide, they overlap in the frequency domain and some spatial frequency components contain the sum of the luminance and chrominance signals instead of each of them separately. In this case, demosaicing algorithms reconstruct luminance information in chrominance and vice-versa, which results in visual artifacts. As illustrated in Figure 5, there are in fact four kinds of reconstruction artifacts possible, two more than described by Topfer et al. [5].
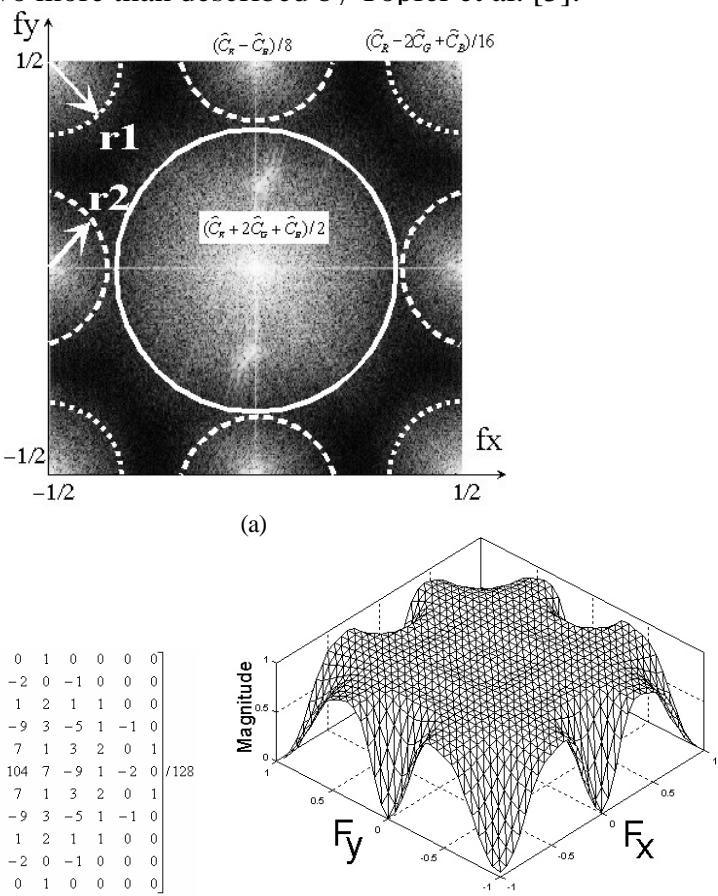

(c)

Figure 4: (a) Fourier representation of a CFA image. The energy of the luminance is concentrated in the center of the image. The energy of chrominance is located on the borders. (b) The luminance filter used in the simulations discussed in Section V. (c) Representation of the transfer function of the luminance estimation filter.

One is the apparent blurring due to the under-estimation of the luminance spectrum, i.e. choosing a too narrow-band spatial low-pass filter. Choosing a filter with a spatially wider band can result in a grid effect especially visible in flat (homogeneous) regions of the image due to the high frequency content of the chrominance signal spilling into luminance, which is the second artifact. The same applies for chrominance. False color appears due to high luminance frequencies in the chrominance signal when the spatial high-pass filter is too wide-band. When the spatial filter band is too narrow, a "watercolor" effect might appear as colors spread beyond the edges of an object. For classical demosaicing algorithms, the two most visible effects are blurring and false color because these algorithms tend to underestimate luminance and overestimate chrominance. But, using more recent methods, grid effects and watercolor can be observed, as is illustrated in several publications [6, $28,38,40]$.

The Fourier representation also helps to explain why the Bayer CFA is the optimal spatial arrangement in terms of spatial frequency representation when placing three colors on a square grid. The sampling period of each color in the CFA is two in the horizontal, vertical and diagonal direction (even one in the diagonal for green), as shown in Figure 1, which carries the chrominance at frequency $1 / 2$ in the horizontal and vertical direction, as shown in Figure 4a. The chrominance and luminance spectra are thus maximally separated because the chrominance energy is located on 
the borders of the Fourier spectrum and luminance in the center, allowing the best distinction between them in the Fourier domain.

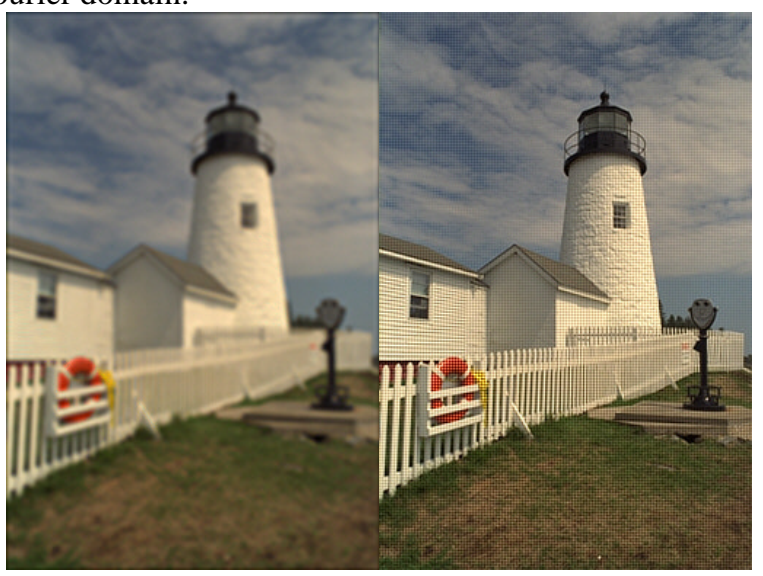

(a)

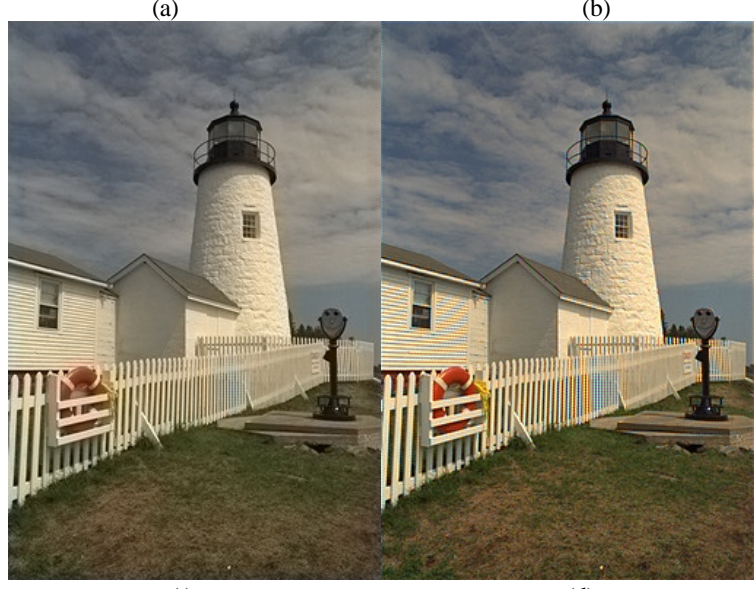

(c)

(d)

Figure 5: The four artifacts visible after demosaicing: (a) Excessive blurring (b) Grid effect (c) Watercolor (d) False color.

If the period is higher, the chrominance spectra would be located closer to the center of the spectrum, increasing the probability of aliasing between luminance and chrominance. The Bayer CFA is the only proposed CFA on a square grid that provides the same horizontal and vertical sampling frequency for each color and a maximum sub-sampling by a factor of two in the horizontal, vertical, and diagonal direction. Other proposed CFAs, such as stripes or interlaced stripes, have at least the horizontal or vertical direction sub-sampled by a factor of three. Also, the fourcolor CFA proposed recently does not provide a reduction of aliasing since its frequency pattern is identical to the Bayer and the addition of a color should reduce correlation between color channels and worsen aliasing by increasing the chrominance bandwidth. However, a CFA on a hexagonal grid should provide better reconstruction of three colors

\section{The new demosaicing algorithm}

In summary, the new algorithm we propose is composed of five steps [3, 4]. First, we estimate the luminance signal from the spatially multiplexed image by low-pass filtering, for example with the filter proposed in Figure 4b. Second, we estimate chrominance by high-pass filtering. This highpass filter can be designed to be orthogonal to the luminance filter. In that case, the chrominance signal is obtained by subtracting the estimated luminance from the CFA image. Third, we de-multiplex the chrominance by multiplying it with the modulation functions given in eq. 7 , which results in three opponent chromatic and sub-sampled signals as shown in eq. 10 and 11 . Fourth, we interpolate the opponent chromatic signals, using for example filters similar to the ones defined in eq. 2 . Fifth, we reconstruct the original image as a sum of luminance and interpolated opponent chromatic signals to provide three color components per pixel.

It should be noted that even though this algorithm has a number of steps, it is efficient because most of the steps have little computational complexity. The most computationally expensive step is the estimation of luminance. For good estimation, a filter of size $11 \times 11$ is usually required. For our proposed filter (Fig. 4b), this results in 69 operations per pixels. The second most computationally expensive step is the interpolation of chrominance. However, since the human visual system is not too sensitive to spatial acuity in chrominance, a simple bilinear interpolation is sufficiently accurate.

\section{LUMINANCE AND CHROMINANCE FILTERS}

We have seen that the reconstruction quality of the demosaicing algorithm depends on the estimation of luminance and chrominance. The luminance should be estimated directly from the CFA image to optimize the reconstruction of achromatic spatial information. A good filter design is a compromise between allotting a large enough spatial bandwidth for luminance and chrominance while reducing as much as possible the mis-categorization of each. Expressing the problem of demosaicing solely in this way, it is impossible to design filters for accurate reconstruction. Even if luminance and chrominance have a well-separated location in the Fourier domain, they have to share the Fourier space for their own representation. If we suppose that they don't alias and that each occupies only half of the Fourier spectrum where they are represented alone, we would have the same condition as in Section II. However, luminance and chrominance do not occupy the same sized area in the Fourier domain. Luminance can be estimated with high spatial frequency, which ensures a good reconstruction of edges.

The three RGB channels are usually correlated along the wavelength dimension because the filter and sensor sensitivities $\varphi_{i}$ generally overlap. In other words, the information captured in one channel might also be present in another one. They are also correlated in space, because neighboring pixels have a large probability to be identical. This spatialchromatic correlation is useful in CFA images even if it is not easy to quantify and control.

There is no formal relationship between correlation and bandwidth. However, if we set a threshold beyond which 
we consider the luminance and chrominance spectra no longer useful, and define the bandwidth from this threshold, we can see that the bandwidth of chrominance is narrower than that of luminance as illustrated in Figure 4-a, where the radii of the circles for chrominance are smaller than the radius for luminance.

We can define an arbitrary filter response as follows. We assume that the chrominance filters have a Gaussian response. The two parameters $\mathrm{r} 1$ and $\mathrm{r} 2$ are the diameters of the Gaussian function centered in the chrominance spectra, as illustrated in Figure 4a. The choice of Gaussian shaped filters is arbitrary. Considering that we have no knowledge of the frequency distribution of the chrominance and luminance signals in a natural image (i.e. the "areas" they occupy in the Fourier spectrum), it gives a good compromise of spatial versus frequency behavior and is practical for FIR filter design. We can then iteratively compute the optimal parameters $\mathrm{r} 1$ and $\mathrm{r} 2$ to result in the best reconstruction in terms of CPSNR (color-peak-signalto-noise-ratio). Note that other error criteria, such as $\Delta \mathrm{E}$, could also be used. Let $I_{1}$ and $I_{2}$ be the original and reconstructed images of height $\mathrm{H}$ and width $\mathrm{W}$, expressed in integer values between 0 and 255 . The CPSNR was calculated as follows:

$$
\begin{aligned}
& C M S E=\frac{1}{3 H W} \sum_{i=1}^{3} \sum_{x=1}^{H} \sum_{y=1}^{W}\left(I_{1}(x, y, i)-I_{2}(x, y, i)\right)^{2} \\
& C P S N R=10 \log _{10}\left(\frac{255^{2}}{C M S E}\right)
\end{aligned}
$$

The resulting luminance filter, optimized for the four images given in Figure 6, is illustrated in Figure $4 \mathrm{~b}$. The resulting transfer function of the filter, illustrated in Figure 4-c, shows that the radius $\mathrm{r} 1$ is larger than $\mathrm{r} 2$ for the images we have tested. This is because the geometry of the Fourier transform of a square grid allows for more 'space' for the chrominance in the corner (i.e. $\hat{C}_{R}-2 \widehat{C}_{C}+\widehat{C}_{B}$ ) than for the chrominance in the middle (i.e. $\widehat{C}_{R}-\widehat{C}_{B}$ ). Consequently, this part of the chrominance should be reduced to allocate more bandwidth to luminance in this region of the Fourier space, which results in better CPSNR.

Intuitively, it would therefore be more appropriate to change the arrangement of color pixels in the Bayer CFA. We can change, for example, the amount and positioning of green and blue pixels. Red and green are usually more correlated than ed and blue; their sensitivity functions overlap more. This would result in reducing the probability of aliasing between chrominance located in the middle and luminance, and allow for a better estimation of the spatial information (because $\widehat{C}_{R}-\widehat{C}_{B}$ will be changed to $\widehat{C}_{R}-\widehat{C}_{G}$, which occupies a smaller bandwidth). An example is given in Table 1 for the four images we have tested (see Figure 6). Note that for this example, we calculated the optimal filters for each image. The reconstruction is systematically better when the green pixels in the Bayer CFA are exchanged by red or blue. Therefore, having more green pixels in a CFA is not a better way to improve the spatial resolution of the demosaiced image, contrary to what it is generally thought. The weights of R, G and B that define luminance should be designed to optimize spatial acuity and not to match as closely as possible the human luminosity function. Visual examples of images mosaiced with the modified Bayer CFA and demosaiced by frequency selection can be found in [43].

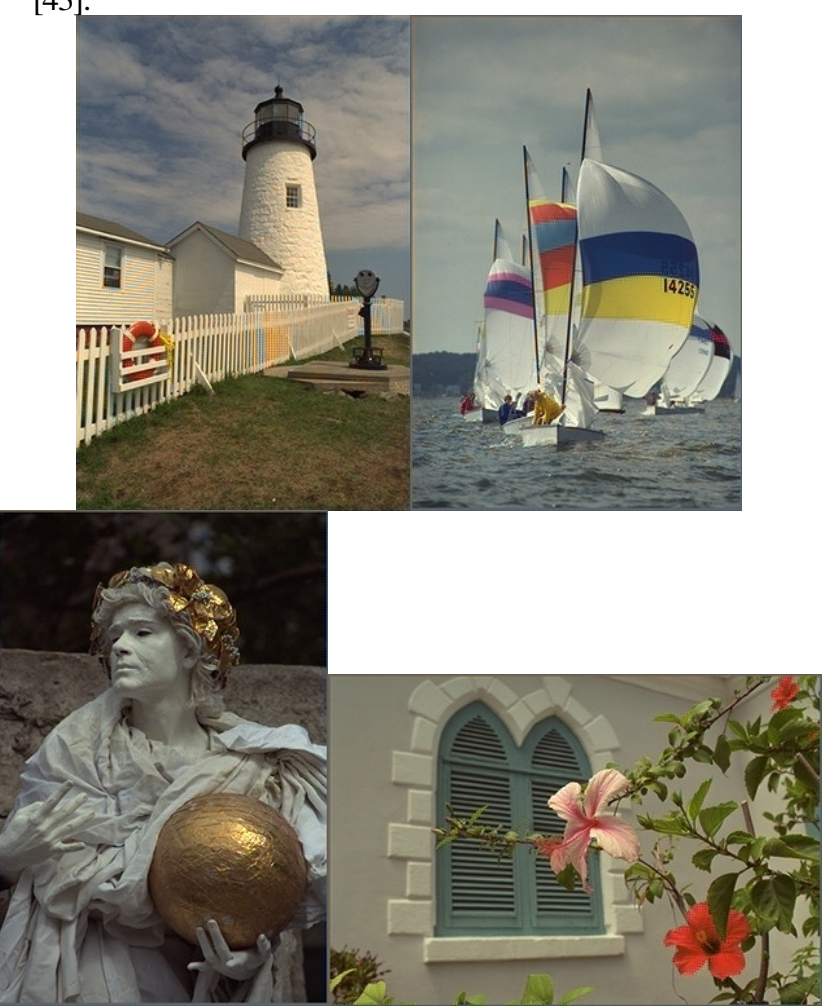

Figure 6: Results of the frequency selection demosaicing algorithm on four images. The same luminance filter (Figure 4-b) is used for the reconstruction.

TABLE 1:

Comparison of CPSNR obtained with the Bayer CFA, a modified Bayer CFA where the red and green pixels are exchanged, and a CFA where the blue and green pixels are exchanged. An optimal filter is computed for each image and each CFA with respect to CPSNR (eq. 15).

\begin{tabular}{|l|l|l|l|}
\hline & $\begin{array}{l}\text { Bayer } \\
\text { CFA }\end{array}$ & $\mathrm{R} \Leftrightarrow \mathrm{G}$ & $\mathrm{B} \Leftrightarrow \mathrm{G}$ \\
\hline Lighthouse & 33.88 & 34.43 & 34.34 \\
\hline Sails & 35.72 & 36.28 & 36.59 \\
\hline Statue & 37.70 & 37.92 & 37.86 \\
\hline Window & 35.27 & 35.47 & 35.87 \\
\hline
\end{tabular}

\section{RESULT AND COMPARISON}

In this section, we review the principal demosaicing algorithms described in the literature. There are many proposed methods, using the entire gamut of signal and image processing algorithms, from signal interpolation, neural networks, Bayesian methods, regularization method and methods specific to color science. It is not possible to discuss all the published methods and compare them with our result, because they are too numerous and the implementations are not always given with enough details. We have selected several of them that we find representative and discuss their relationship with our model 
of CFA images. We also compare the performance of some of them with the performance of our demosaicing algorithm.

\section{A. State of the art}

The first approach to improve bilinear interpolation results, which exploits correlation, is based on the concept of computing hue as the ratio of two colors 9]. The underlying image model assumes that the correlation between color channels implies that the red and blue pixels are additively related to the green pixels, and an estimate of the red and blue measurements can be obtained by adding a constant to green. Assuming that hue does not change over the surface of an object, it is more reliable to interpolate color ratios instead of R, G, and B separately in terms of avoiding false color. This approach gives better results than bilinear interpolation for a reasonable increase of computation time, as shown in Table 2. However, artifacts are still visible around the border of an object or in textured regions where the assumption of constant hue does not hold.

To overcome the problem of object contours, Cok $[17,18]$ has proposed an improved interpolation method that takes the edges in the image into account. This approach has been generalized to an adaptive interpolation method that is also called template matching. The type of contour is classified and the interpolation changes according to the contour type. Cok $[9,18]$ has proposed to classify the interpolation patterns into three classes: edge, stripe and corner (a review is given in [19]). The different interpolations, based on median filtering, are applied dependent on the type of neighborhood of the interpolation point. Several authors [20-27, 42] have proposed such adaptive algorithms. These methods differ not only in the way the image template is estimated (either based on convolution kernels, image gradients or median filtering), but also in the interpolation procedure applied. The advantage of these algorithms is that they adaptively interpolate following the local content of the image and result usually in improved quality compared to nonadaptive algorithms. However, the computation time is image dependent and often longer. Improving these algorithms by taking into account more neighboring patterns results in higher computational complexity [27]. A way to avoid image dependence is to use an edge function computed from the image gradient [28-29]. This edge function is then included in the bilinear interpolation, changing the relative weight of each neighboring pixel, following the intensity of the edge. The advantage of such an approach over the adaptive methods is that edges are only computed once and known exactly from the existing pixels.

Some authors consider a CFA image to be threedimensional in the sense that two dimensions contain spatial and one wavelength information, and propose interpolation algorithms for this sub-sampled threedimensional space. Gupta et al. [31] propose to apply median filtering, with the vectors composed of the three colors' neighboring pixels. Other authors [32-35] have employed this paradigm in more sophisticated ways. Jinwo ok et al. [34] propose the use of neural networks. Trussel et al. [33] use optimal wiener filtering. These techniques allow adding some knowledge of the physical process of sampling to the optimization. Taubman [32] proposes a de-convolution of the optical blur conjointly with the demosaicing algorithm. Unfortunately, these algorithms are application dependent and need to be optimized for each specific camera.

Some attempts at generalization have been proposed, such as using Bayesian theory where the knowledge consists of an ad-hoc function [38]. Also, Muresan et al. [30] propose to build a metric from a training set to define the interpolation class. In general, we would call these approaches "de-aliasing methods," because they use knowledge about the image formation process. Iterative methods such as in Kimmel [28], Gunturk et al. [40] for color correction and Keren et al. [35] for regularization could also be classified in this category. As a metric defined from a training set, iteration procedures imply pior knowledge because one supposes that there will be a solution that converges.

TABLE 2:

Comparison of CPSNR (eq. 15) and computation time between differen demosaicing algorithms. The same luminance filter (Figure 4-b) is used for all images demosaiced by frequency selection.

\begin{tabular}{|l|c|c|c|c|c|c|}
\hline & Bilinear & $\begin{array}{c}\text { Hue } \\
\text { Based }\end{array}$ & $\begin{array}{c}\text { Gradient } \\
\text { Based }\end{array}$ & $\begin{array}{c}\text { Alternating } \\
\text { projection 1 }\end{array}$ & $\begin{array}{c}\text { Alternating } \\
\text { projection 2 }\end{array}$ & $\begin{array}{c}\text { Frequency } \\
\text { Selection }\end{array}$ \\
\hline Lighthouse & 25.44 & 27.04 & 31.5 & 32.33 & 35.26 & 33.75 \\
\hline Time & 1 & 1.70 & 49.59 & 28.34 & 32.64 & 2.30 \\
\hline Sails & 28.43 & 29.91 & 34.37 & 34.89 & 36.92 & 35.72 \\
\hline Time & 1 & 1.65 & 48.21 & 28.34 & 32.47 & 2.30 \\
\hline Statue & 28.36 & 29.66 & 32.78 & 35.86 & 37.65 & 37.53 \\
\hline Time & 1 & 1.34 & 48.21 & 29.75 & 33.94 & 2.41 \\
\hline Window & 27.96 & 29.60 & 31.46 & 34.76 & 35.40 & 34.79 \\
\hline Time & 1 & 1.31 & 48.87 & 27.99 & 32.08 & 2.31 \\
\hline
\end{tabular}

In general, reconstruction methods based on interpolation are predominant. They result in efficient algorithms that can be extended by other signal processing techniques. Adams [7] has studied an approximation of the ideal filter, taking into account the neighboring pixels of different color. He found that a second derivative of the Laplacian, applied on other colors than those interpolated, act as an interpolation corrector. Bongjun et al. [36] propose the use of splines to improve the bilinear interpolation filter. Goltzbach et al. [6] use specific two-dimensional filters to add the high frequency components of the green channel to the red and blue. Weerasinghe et al. [37] consider the green channel separately. Methods using bilateral filtering [39], a non-linear generalization of the edge-based algorithms, have also been studied. And finally, it is also possible to track the local properties of the image using wavelet decomposition [41], which allows for optimizing the correlation in spatial and frequency domain.

A recent method by Gunturk et al. [40] using wavelet decomposition to allow exchanging high spatial frequencies of red and blue with green (as previously proposed in [6]), using template matching [42] as initialization and an iterative process called "alternating projection" for 
correction results currently in the best quality reconstructions that we are aware of. Our method, using linear filtering, also performs well and is computationally more efficient.

\section{B. Comparison}

We compared our demosaicing algorithm to four other methods: bilinear interpolation as described in eq. 2, constant hue [9], gradient based [28], and alternating projection [40] with either (1) bilinear interpolation or (2) template matching [42] as initialization, one level of decomposition, and eight iterations. We found that our method performed well for four images of size $384 \times 256$ pixels taken from the Kodak database. We compared CPSNR and computational time, assuming bilinear interpolation to be 1 time unit.

The reconstructed images are shown in Figure 6, with more detailed images and comparisons on our web site [43] The results are listed in Table 2. Our demosaicing by frequency selection method results in good CPSNR and good visual quality for a very short computation time compared with algorithms resulting in similar quality. The reconstruction of all four images uses the luminance estimator given in Figure 4b. For an illustration of the images using other demosaicing algorithms, see references $[23,27,28,40]$ that contain comparative studies for the same images.

\section{CONCLUSION}

We have developed a Fourier-domain model for spatial multiplexing of color, common to CFA images and the human retina. We demonstrate that a one-color per pixel image is equivalent to a sum of luminance and sub-sampled and modulated opponent chromatic signals. In the case of a regular CFA arrangement, such as the Bayer CFA, luminance and chrominance are well localized in the spatial frequency domain. They can be estimated by appropriate frequency selection $[3,4]$. We also describe a method for designing a luminance estimation filter based on the estimation of the scalar luminance directly from CFA images. Additionally, we have explained the artifacts generated by the reconstruction of the image as aliasing between luminance and chrominance. These artifacts are common to all demosaicing algorithms. We can also use this representation to design optimal selection filters that provide a good and efficient demosaicing method.

This model also shows that the Bayer CFA is the most optimal spatial arrangement of three colors on a square grid. However, if we additionally take into account the correlation between color channels, the Bayer CFA should be modified to a CFA having two times more blue pixels than red and green. Such an arrangement allows estimating achromatic spatial acuity to higher frequencies.

Our demosaicing algorithm based on frequency selection performs well compared to other methods. It provides a good compromise between quality of reconstruction and computational complexity, because it can be implemented by linear filtering. Moreover, this algorithm could be extended to include some properties of previously proposed algorithm, such as adaptive interpolation or interpolation based on prior knowledge of color images. Identically, the frequency properties of a CFA image allow designing an improved interpolation in an explicit and controllable way.

There still remain two main extensions that have not yet been addressed. First, a better understanding of the spatial and chromatic statistical properties of natural color images could certainly help in the design of optimal luminance and chrominance filters. Second, it could be interesting to consider the random sampling properties of the human visual system as a model for allowing alias-free reconstruction of high spatial acuity, and to investigate how it applies to digital imaging.

\section{ACKNOWLEDGMENT}

The athors thank the anonymous reviewers for their comments, which substantially improved the manuscript. We also thank Antonio Torralba and Jean-Marc Vesin for fruitful discussions.

\section{REFERENCES}

[1] K.T. Mullen, "The contrast sensitivity of human colour vision to redgreen and blue-yellow chromatic grating," Journal of Physiology, 359, 1995, pp. 381-400.

[2] D.R. Williams, N. Sekiguchi, W. Haake, D. Brainard and O. Packer, "The cost of trichromacy for spatial vision," From pigment to perception, A. Valberg and B.B. Lee, ed, Plenum Press, New York, 1991, pp. 11-22.

[3] D. Alleysson and J. Hérault, "Interpolation d'images couleurs sous échantillonnées par un modèle de perception [Sub-sampled color image interpolation by a perception model]," Proc. GRETSI'2001, Toulouse, 2001

[4] D. Alleysson, S. Süsstrunk, and J. Hérault, "Color Domosaicing by estimating luminance and opponent chromatic signals in the Fourier domain," Proc. IS\&T/SID Color Imaging Conference, Scottsdale, 2002, pp. 331-336.

[5] K. Topfer, J.E. Adams, and B.W. Keelan, "Modulation transfe functions and aliasing patterns of CFA interpolation algorithms," Proc. IS\&T PICS, Portland Oregon, 1998, pp. 367-370

[6] J.W. Glotzbach, R.W. Schafer, and K. Illgner, "A method of color filter array interpolation with alias cancellation properties," Proc. Int'l Conf. on Image Processing, (ICIP'01), Thessaloniki, Greece, vol. 1, Oct. 2001, pp. 141-144.

[7] J.E. Adams Jr., “ Design of practical color filter array interpolation algorithms for digital cameras II," Proc. Int'l Conf. Image Processing, (ICIP'98), Chicago, IL, USA, vol. 1, Oct. 1998, pp. 488-492.

[8] J. Greivenkamp, "Color dependant optical prefilter for the suppression of aliasing artifacts," Applied Optics, vol. 29, no. 5, 1990, pp. 676-684.

[9] D.R. Cok, "Reconstruction of CCD Images Using Template Matching," Proc. IS\&T's 47th Annual Conference and Int'l Congress Photo. Sciences (ICPS'94), 1994, pp. 380-385.

[10] J.A. Weldy, "Optimized design for a single-sensor color electronic camera system," SPIE Optical sensors and Electronic Photography, vol. 1071,1989 , pp. 300-308.

[11] B.E. Bayer, Color imaging array, US Patent 3,971,065, to Eastman Kodak Company, Patent and Trademark Office, Washington, D.C., 1976.

[12] J.I. Yellott Jr., "Spectral analysis of spatial sampling by photoreceptors: Topological disorder prevents aliasing”, Vision Research, vol. 22, 1982, pp. 1205-1210. 
[13] G. Buchsbaum and A. Gottschalk, "Trichromacy, opponent colours coding and the optimum colour information transmission in the retina", Proc. R.. Soc. Lond., B220, 1983, pp. 89-113.

[14] C.R. Ingling Jr. and E. Martinez, "The spatiochromatic signal of the rg channel," Colour Vision, J.A. van Esch et al., ed., Academic Press London, 1983.

[15] H.D. Crane, J.D. Peter, and E. Martinez-Uriegas, Method and Apparatus for Decoding Spatiochromatically Multiplexed Color Images Using Predetermined Coefficients, US patent 5,901,242, to SRI International, Patent and Trademark Office, Washington, D.C., 1999.

[16] D.R. Cok, Signal Processing Method and Appartus for Producing Interpolated Chrominance Values in a Sampled Color Image Signal, US patent 4,642,678, to Eastman Kodak Company, Patent and Trademark Office, Washington, D.C., 1987.

[17] D. R. Cok, Method of processing sampled signal valves produced by a color imaging device, US patent 5,040,064, to Eastman Kodak Company, Patent and Trademark Office, Washington, D.C., 1991.

[18] D.R. Cok, Signal processing method and apparatus for sampled image signals, US patent 4,630,307, to Eastman Kodak Company, Patent and Trademark Office, Washington, D.C., 1986.

[19] J.E. Adams Jr, "Interactions Between Color Plane Interpolation and Other Image Processing Functions in Electronic Photography," Proc. SPIE, Cameras and Systems for Electronic Photography and Scientific Imaging, ed. C.N. Anagnostopoulos/M.P. Lesser, vol. 2416, 1995, pp. 144-151.

[20] C.A. Laroche and M. A. Prescott, Apparatus and method for adaptively interpolating a full color image utilizing chrominance gradients. US patent 5,373,322, to Eastman Kodak Company, Patent and Trademark Office, Washington, D.C., 1993.

[21] J.E. Adams Jr. and J.F. Hamilton, Jr., Adaptive color plane interpolation in single sensor color electronic camera, US patent 5,652,621, to Eastman Kodak Company, Patent and Trademark Office, Washington, D.C., 1997.

[22] E. Chang, S. Cheung, and D. Pan, "Color filter array recovery using a threshold-based variable number of gradients," IS $\backslash \&$ T's Conference on Sensor, Camera and Applications for Digital Photography, San Jose California, vol. 3650, 1999, pp. 36-43.

[23] T. Kuno and H. Sugiura, "New interpolation method using discriminated color correlation for digital still cameras," IEEE Transaction on Consumer Electronics, vol. 45, no. 1, Feb. 1999, pp 259-267.

[24] J.F. Hamilton Jr. and J.E. Adams Jr., Computing Color Specification (Luminance and Chrominance) Values for Images, US patent 6,075,889, to Eastman Kodak Company, Patent and Trademark Office, Washington, D.C., 2000.

[25] T. Acharya, Efficient algorithm for color recovery from 8bit to 24-bit color pixels, US patent 6,091,851, to Intel Corporation, Patent and Trademark Office, Washington, D.C., 2000.

[26] P. Soo-Chang and T. Io-Kuong, "Effective color interpolation in CCD color filter array using signal correlation," Proc. Int'l Image Processing, (ICIP'00), IEEE CS Press, Vancouver, BC, Canada, vol. 3, 2000, pp. 488-491.

[27] W. Lu and Y.P. Tan, "Layering-based color filter array interpolation," Proc. Int'l Conf. on Image Processing, Thessaloniki, Greece, vol.3, Oct. 2001, pp. 860-863

[28] R. Kimmel, "Demosaicing: Image Reconstuction from Color Samples," IEEE Trans. Image Processing, vol. 8, Sept. 1999, pp. 1221-1228.

[29] X. Li and M. Orchard, "New edge-directed interpolation," IEEE Transactions on Image Processing, vol. 10, no. 10, Oct. 2001, pp. 1521 1527.

[30] D. Muresan and T. Parks, "Optimal recovery demosaicing," IASTED Signal and Image Processing, Hawaii, 2002.

[31] M. Gupta and T. Chen, "Vector Color Filter Array Demosaicing," Proceedings of the SPIE, Sensors and Camera Systems for Scientific, Industrial, and Digital Photography Applications II, Vol. 4306, San Jose, ed, CA, M. Blouke, J. Canosa, N. Sampat, Jan. 2001, pp. 374 382.

[32] D. Taubman, "Generalized Weiner reconstruction of images from colour sensor data using a scale invariant prior," Proc Int. Conf. Image Proc., (ICIP’00), Vancouver, BC, Canada, 10-13 Sept. 2000, vol.3, pp. 801804.
[33] H. Trussell and R.E. Hartwig, "Mathematics for demosaicing," IEEE Transanctions on Image Processing, vol. 11, no 4, April 2002, pp. 495492.

[34] G. Jinwook, S. Kwanghoon, and L. Chulhee, "Interpolation using neural networks for digital still cameras," IEEE Trans. on Consumer Electronics, vol. 46 no 3, Aug. 2000, pp. 610-616.

[35] D. Keren and M. Osadchy, "Restoring subsampled color images," Machine Vision and Applications, ed. Springer-Verlag, vol. 11, 1999, pp197-202.

[36] L. Bongjun, S. Kwanghoon, and L. Chulhee, "Performance comparison of various interpolation methods for color filter arrays," Proc. Int'l Symposium on Industrial Electronics (ISIE'01), Pusan, South Korea, June 2001, vol. 1, pp. 232-236.

[37] C. Weerasinghe, I. Kharitonenko, and P. Ogunbona, "Method of color interpolation in a single sensor color camera using green channel separation," Proc. Int'l Conf. on Acoustics, Speech, and Signal Processing, (ICASSP'02), May 13-17, 2002, vol. 4, pp. 3233-3236.

[38] D.H. Brainard, "Reconstructing Images from Trichromatic Samples, from Basic Research to Practical Applications," Proc. IS\&T/SID 3rd Color Imaging Conference, (CIC'95), Scottsdale, Arizona, 1995, pp. 4 10.

[39] R. Ramanath and W.E. Snyder, "Adaptive Demosaicking," Journal of Electronic Imaging, vol. 12, no. 4, Oct. 2003, pp. 633-642.

[40] B.K. Gunturk, Y. Altunbasak, and R.M. Mersereau, 'Color plane interpolation using alternating projections,"IEEE Transactions on Image Processing, vol. 11, no 9, Sept. 2002, pp. 997-1013

[41] B. Tao, I. Tastl, T. Cooper, M. Blasgen, and E. Edwards, "Demosaicing using human visual properties and wavelet interpolation filtering", Proc. IS\&T/SID Seventh Color Imaging Conference, (CIC'99), Scottsdale, Arizona, 1999, pp.252-256.

[42] J.F. Hamilton Jr. and J.E. Adams, "Adaptive color plane interpolation in single sensor color electronic cameras," U.S. Patent 5,629,734, to Eastman Kodak Company, Patent and Trademark Office, Washington, D.C., 1997.

[43] Additional figures are available at: http://ivrgwww.epfl.ch/index.php?name=IEEE Demosaicing

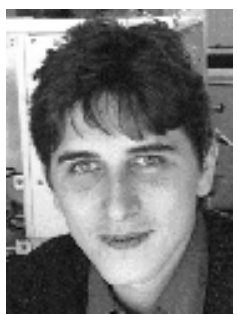

David Alleysson was born in France. He received his B.S. degree in Computer Science from the Ecole d'Ingénieurs de Genève, Geneva in 1994, and his M.S. degree in Computer Science from ENSIMAG (Ecole National Supérieure d'Informatique et Mathématique de Grenoble) in 1995. He received his $\mathrm{PhD}$ from the Université Joseph Fouier in Computer Science, option Cognitive Science, in 1999.

$\mathrm{He}$ is currently research associate at CNRS. His current research interests are the neurobiological foundations of vision and color vision and its applicability to image processing and image representation.

Dr. Alleysson is a member of IS\&T and ICVS.

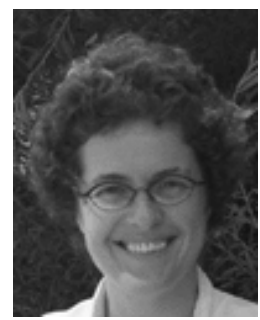

Sabine Süsstrunk (M'02) is Assistant Professor for Images and Visual Representation at the Ecole Polytechnique Fédérale de Lausanne (EPFL), Switzerland.

She received her B.S. degree in Scientific Photography from the Swiss Federal Institute of Technology in Zurich, Switzerland, in 1987, and her M.S. degree in Graphic Arts Publishing, concentration electronic publishing, from the Rochester Institute of Technology, USA, in 1993. She is currently pursuing a Ph.D. in Computer Science part-time at the University of East Anglia, Norwich, UK. Prof. Süsstrunk is a member of IS\&T and OSA. 


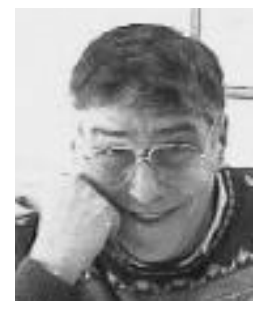

Jeanny Hérault received the M.S. degree in electronics engineering in 1966 at ENSERG, Docteur-Ingénieur and Docteur ès Sciences degrees in 1974 and 1980 from Institut National Polytechnique of Grenoble. Since 1984, he is full Professor at University Joseph Fourier of Grenoble. From 1985 to 1994, he has also been the director of the "Institut des Sciences et Techniques" now POLYTECH'G.

Since 2003, he heads with Alain Lecomte the Master Cognitive Science.

Prof Hérault is presently interested in models of visual perception and high dimensional data processing through selforganizing neural networks. 